Chapman University

Chapman University Digital Commons

Pharmacy Faculty Articles and Research

School of Pharmacy

$3-2008$

\title{
Insights into Morphological Nature of Precipitation of Cholesterol
}

Vuk Uskoković

Chapman University, uskokovi@chapman.edu

Follow this and additional works at: http://digitalcommons.chapman.edu/pharmacy_articles

Part of the Cardiovascular Diseases Commons, and the Medical Biochemistry Commons

\section{Recommended Citation}

Uskoković V. Insights into morphological nature of precipitation of cholesterol. Steroids. 2008;73(3):356-369. doi: 10.1016/ j.steroids.2007.12.005

This Article is brought to you for free and open access by the School of Pharmacy at Chapman University Digital Commons. It has been accepted for inclusion in Pharmacy Faculty Articles and Research by an authorized administrator of Chapman University Digital Commons. For more information,

please contact laughtin@chapman.edu. 


\section{Insights into Morphological Nature of Precipitation of Cholesterol}

Comments

NOTICE: this is the author's version of a work that was accepted for publication in Steroids. Changes resulting from the publishing process, such as peer review, editing, corrections, structural formatting, and other quality control mechanisms may not be reflected in this document. Changes may have been made to this work since it was submitted for publication. A definitive version was subsequently published in Steroids, volume 73, issue 3, in 2008. DOI: $10.1016 /$ j.steroids.2007.12.005

The Creative Commons license below applies only to this version of the article.

\section{Creative Commons License}

\section{(c) (1) 9}

This work is licensed under a Creative Commons Attribution-Noncommercial-No Derivative Works 4.0 License.

\section{Copyright}

Elsevier 


\title{
Insights into Morphological Nature of Precipitation of Cholesterol
}

\author{
Vuk Uskoković \\ Center for Advanced Materials Processing, Clarkson University, Potsdam, NY, USA
}

\begin{abstract}
Additional effects on the previously reported procedure of precipitation of narrowly dispersed and well-defined, brick-shaped cholesterol particles, including nonsolvent addition rate, temperature, solvent purity, aging treatments, ultrasound agitation and fine mechanical effects were investigated. Based on the presented results, significant morphological sensitivity of cholesterol precipitation processes upon variations from the standard established procedure of crystallization is induced. However, the tendency of cholesterol to crystallize in form of biaxially grown particles was evidenced as dominating the precipitation processes, irrespective of any modifications of experimental parameters involved in the preparation procedure investigated hereby. Prolonged aging time and temperature effects lead to "face-to-face" aggregation of particles, promoted by the discrepancy in surface charges between particle sides and faces. In light of these observations, the mechanism of precipitation of cholesterol is further discussed.
\end{abstract}

\section{Introduction}

Cholesterol presents one of the essential biochemical compounds in the animal world, and the actual interest in its physico-chemical and biological characteristics is in large extent instigated by numerous evidenced unbalanced metabolic occurrences that involve its undesired in vivo precipitation. Cholesterol is involved in many biofunctional tasks, including its most notable roles as the precursor for in vivo synthesis of bile acids and steriod hormones ${ }^{1}$, and a regular component of cellular membranes that maintains their flexibility and proper transport properties ${ }^{2}$. However, its pathological crystallization within an organism, supported by a discrepancy between the levels of cholesterol intake and internal production and the rate of its solubilization through the action of micellar, vesicular and bilayer cleansing agents in bile and specific lipoprotein complexes in blood, relates to a wide array of problematic health issues that range from gallstone formation to chronic intestinal lumen deposits to atherosclerotic plaque. Because of the potential development of reversible control, morphological studies of crystallization of cholesterol in simple environments may provide significant relationships for both the areas of biomedicine and chemotherapeutics, and the fundamental understanding of the complex biochemistry of life.

Similar to other 3-hydroxy steroids and their hydrates, cholesterol crystallizes in form of a double-layer structure with an end-for-end arrangement of approximately parallel molecules ${ }^{3}$. Biaxial growth of cholesterol crystals, moreover, results from a faster growth within the bilayer plane comparing to the growth between bilayers, induced by stronger molecular interactions between neighboring molecules in the same bilayer plane ${ }^{4}$. Cholesterol molecules in both in vivo and in vitro conditions, therefore, typically 
crystallize in form of either plate- or needle-shaped particles. Despite the fact that the effects of solvent type ${ }^{5,6,7}$, non-solvent phases ${ }^{8}$, temperature ${ }^{5}, \mathrm{pH}^{9}$, electrolytes ${ }^{10}$, the dynamics of solvent systems ${ }^{11}$, magnetic field ${ }^{12}$, mineral ${ }^{13}$ and polypeptide ${ }^{14}$ substrates and co-existing phases ${ }^{15,16}$ (such as hydroxyapatite deposits, often found interspersed within cholesterol layers in atherosclerotic plaques ${ }^{17,18}$ ), model bile composition ${ }^{19,20,21}$, various medicinal plants ${ }^{22}$ and synthetic biochemical compounds (including phospholipids $^{23}$, cholic acid ${ }^{24}$ and other sterols ${ }^{25}$ ) on the processes of crystallization and dissolution of cholesterol were previously acknowledged, structural and functional flexibility of cholesterol, respectively reflected on the observed variations in crystal surface polarity depending on the solvent character ${ }^{23}$ and the biological role that includes cell membrane flexibility mediation and transmembrane signal messaging ${ }^{2}$, seems to defy all the imposed environmental effects and force the formation of biaxially grown, platelet and needle-type morphologies.

\section{Experimental part}

The procedure of synthesis of narrowly dispersed and well-defined cholesterol particles proceeded as follows ${ }^{26}$. Twenty milligrams of commercial cholesterol were dissolved in $10 \mathrm{ml}$ of 1-propanol. Upon the addition of $10 \mathrm{ml}$ of water, white precipitate forms, but dissolves upon mild shaking. The following $5 \mathrm{ml}$ of water is abruptly poured into the solution, resulting in the formation of a bluish white dispersion, which after one minute of aging turns completely white. After 10 minutes of aging, a few drops of the suspension were deposited onto an SEM sample carrier, dried in air and analyzed. Throughout the whole procedure, the beaker was kept still, without any stirring or ultrasound treatment applied. Morphologies of the prepared cholesterol particles were examined with using scanning electron microscopy (SEM; JEOL JSM-6300) and fieldemission scanning electron microscopy (FE-SEM; JEOL JSM-7400F). Crystallinity of cholesterol powders was investigated with using X-ray diffractometry (XRD; Bruker D8). Vibrational spectra of cholesterol particles were evaluated by means of FourierTransformed Infrared (FT-IR; Mattson Galaxy 2020) spectrometric analysis. Differential scanning calorimetry (DSC; Perkin Elmer DSC 7) measurements were performed in air, applying the scanning rate of $5{ }^{\circ} \mathrm{C} / \mathrm{min}$.

\section{Results and discussion}

A drastic morphological difference between the commercial cholesterol sample and cholesterol particles re-crystallized in accordance with the procedure described in the experimental section, may be distinguished from Fig.1. Whereby the commercial sample comprises rod- and needle-shaped cholesterol crystals, extending over 100 microns in length, the recrystallized sample consists of narrowly dispersed, brick-shaped particles. 


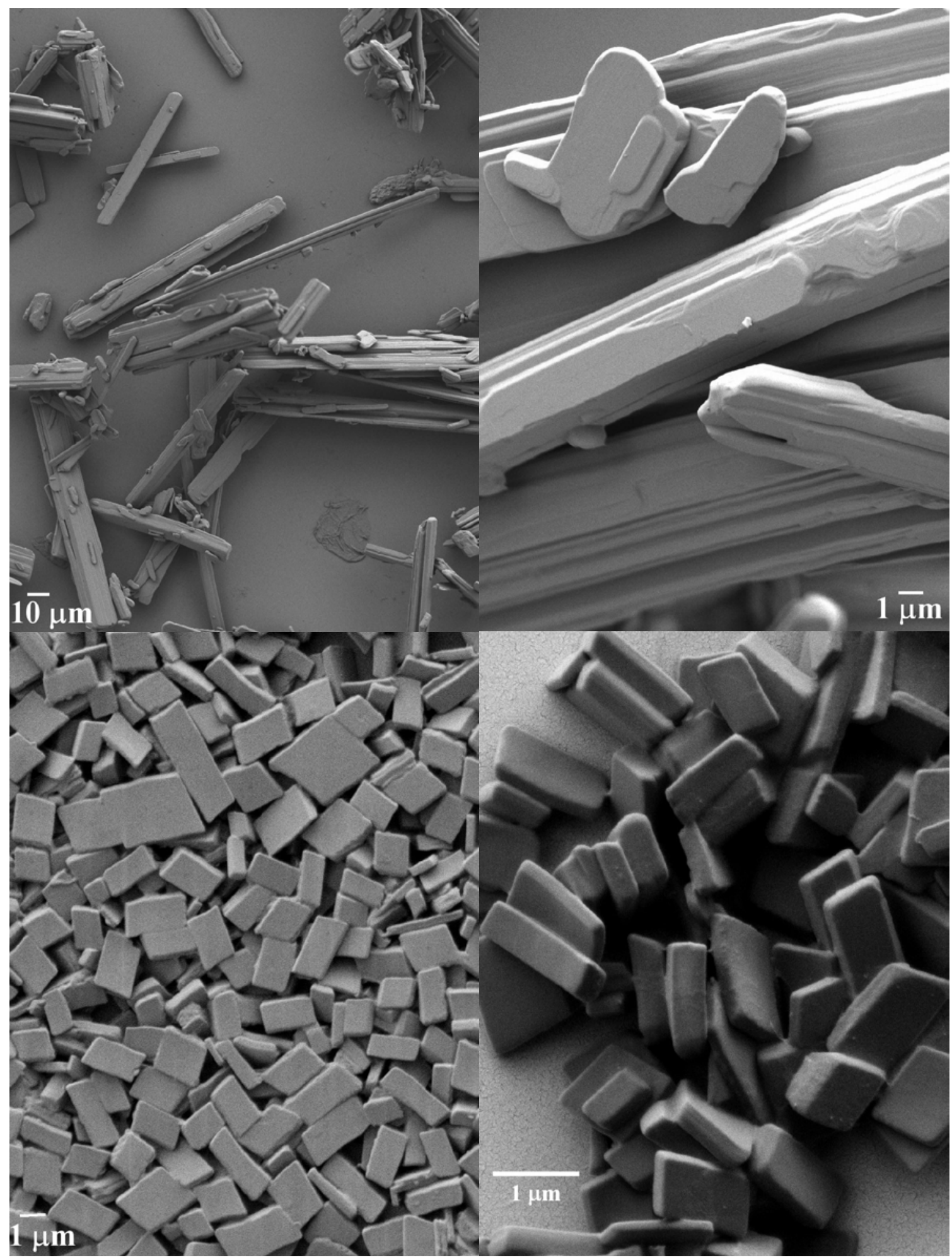

Fig.1. FE-SEM images of the commercial cholesterol (top), and of uniform cholesterol particles precipitated from 1-propanol/water solution, with (bottom left) and without (bottom right) the goldsputtering treatment prior to SEM observations.

DSC, XRD and FT-IR diagrams of both the commercial cholesterol and narrowly dispersed cholesterol powders prepared in 1-propanol/water solutions are successively 
shown in Figs.2-4. Anhydrous modification of the commercial cholesterol may be confirmed through evaluation of the corresponding DSC diagram. Namely, non-existence of the reversible endothermic phase transitions at $\sim 85$ and $120{ }^{\circ} \mathrm{C}$ (which respectively correspond to dehydration effects and the transition from crystalline to liquid crystalline form, typical for cholesterol monohydrate), and the presence of the polymorphic phase transition at $39{ }^{\circ} \mathrm{C}$ are characteristic of anhydrous modification of cholesterol. Furthermore, anhydrous cholesterol typically adopts needle-shaped morphologies, whereas monohydrate modification crystallizes in form of platelets ${ }^{25}$. Elongated morphologies of the crystals that comprise the commercial cholesterol present, therefore, another indication of anhydrous modification of the precursor compound. Previously, it has been detected that the anhydrous form of cholesterol is relatively stable at atmospheric conditions, and that only in the presence of a sufficient amount of water the transition to monohydrate form could be initiated ${ }^{27}$. The polymorphic transition at $39{ }^{\circ} \mathrm{C}$ was detected in case of the re-crystallized sample as well, although with significantly weaker intensity, which might be expected from the fact that mixed polar/non-polar, nonsolvent/solvent system was applied as re-crystallization medium. Whereas cholesterol crystals precipitated in purely alcoholic media are typically characterized by their anhydrous nature, the ones formed in aqueous media are usually monohydrate modification. As a matter of fact, it has been evidenced that 3-hydroxyl groups terminate monohydrate plate faces grown in aqueous environments, whereas alkyl groups predominantly terminate surface of cholesterol crystals grown in anhydrous, alcoholic solutions ${ }^{4}$. Therefore, the pronounced uniaxial growth in case of the crystals grown in anhydrous conditions may be explained by the lack of attachment of water molecules at $\mathrm{OH}$ ends of molecules along (002) plane, that otherwise blocks crystals from growth beyond certain limits in the most favorable direction, which is the one that links (001) and (002) planes and corresponds to the growth along the longest axis of cholesterol molecules. However, similar to the correspondence between plate-shaped crystals and monohydrate form, and needle-shaped crystals and anhydrous modification, respectively, this rule also presents the one with verified exceptions ${ }^{6}$. The most intensive, reversible endothermic peak at $\sim 150{ }^{\circ} \mathrm{C}$ detected in both samples presents the melting point of cholesterol.

There were no significant differences detected between the XRD patterns of the commercial and re-crystallized sample. Both XRD patterns exhibit a pair of characteristic peaks for cholesterol crystals at $2 \theta=2.6$ and $5.2^{\circ}$, corresponding to interfacial, Bragg distances of $d=33.4$ and $16.7 \AA$, as derived from bilayer and monolayer reflections, respectively. The following peaks were detected on both of the cholesterol FT-IR spectra ${ }^{22}$. The peaks at 650,800 and $1060 \mathrm{~cm}^{-1}$ are respectively ascribed to $\mathrm{C}-\mathrm{OH}$ vibration in plane, methylene rock, and C-O stretching vibrations. The peaks at 1260 and $1300 \mathrm{~cm}^{-1}$ respectively correspond to C-H out-of-plane and C-H in-plane bends, whereby the symmetric and asymmetric bends of $\mathrm{CH}$ group are detected at 1370 and $1490 \mathrm{~cm}^{-1}$, respectively. The peak at $1700 \mathrm{~cm}^{-1}$ derives from $\mathrm{C}=\mathrm{C}$ stretching vibration. The peaks in the range of $2600-2900 \mathrm{~cm}^{-1}$ belong to olefinic C-H and alkyl C-H stretching region, whereby the broad absorption band that corresponds to $\mathrm{OH}$ stretching range of frequencies is detected in the range of $3100-3700 \mathrm{~cm}^{-1}$. The only obvious difference between the two cholesterol samples lies in the shape of this broad band. Namely, singletto-doublet transition in the structure of this band is observed as a result of the 
morphological transition from widely dispersed needle-shaped particles of commercial sample to more refined, narrowly dispersed and individual nature of the particles precipitated in 1-propanol. This transition may reflect the increase in structural ordering that corresponds to the formation of monodisperse particles. Because hydrogen bonds provide intermolecular ordering at single-layer structural levels, any increase in structural and morphological symmetry would be reflected on the spatial distribution of $\mathrm{OH}$ bonds and the corresponding distribution of $\mathrm{OH}$ vibrations in IR spectra. The observed symmetry breaking and single-to-doublet transition may, therefore, correspond to an increased symmetry and directional character of the inherent hydrogen bonds (comparing to their more randomly oriented character within the commercial sample) that follows the transition from widely to narrowly dispersed cholesterol particles in terms of their shapes and sizes.

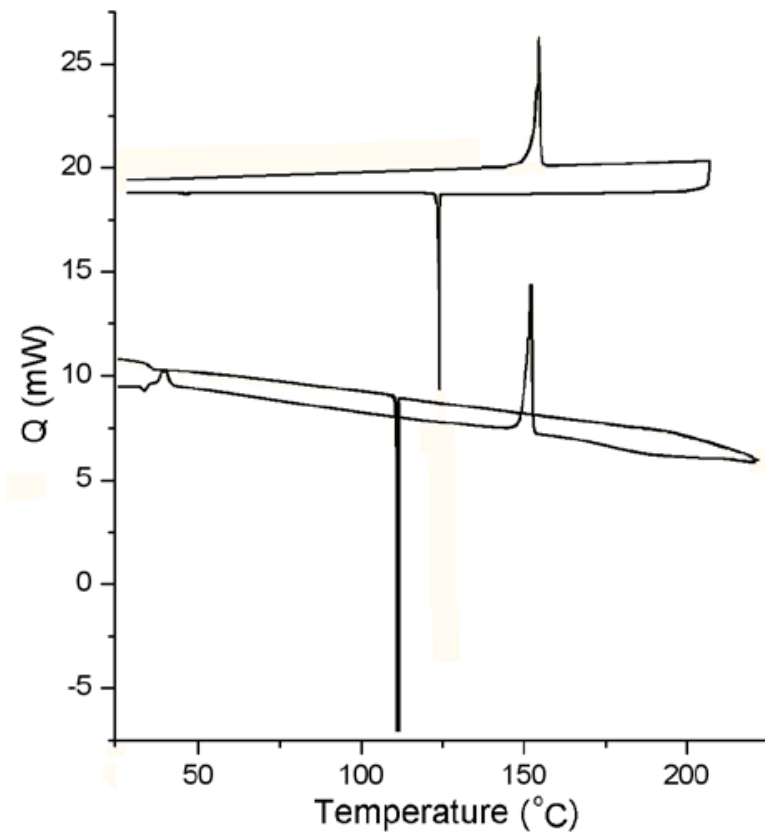

Fig.2. DSC diagrams of the commercial cholesterol (bottom), and of uniform cholesterol particles prepared in 1-propanol/water solution (top).

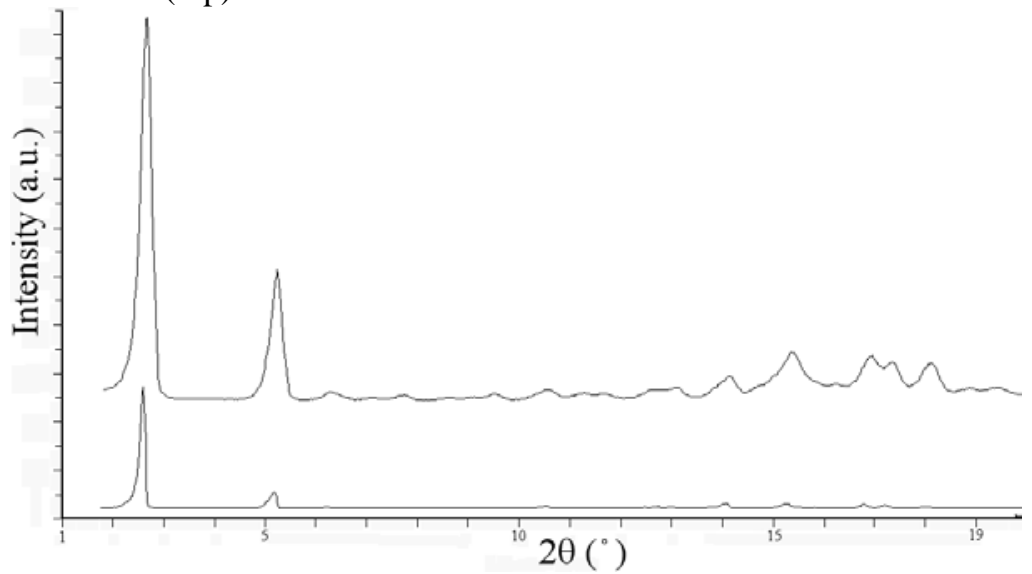

Fig.3. XRD patterns of the commercial cholesterol (bottom), and of uniform cholesterol particles prepared in 1-propanol/water solution (top). 


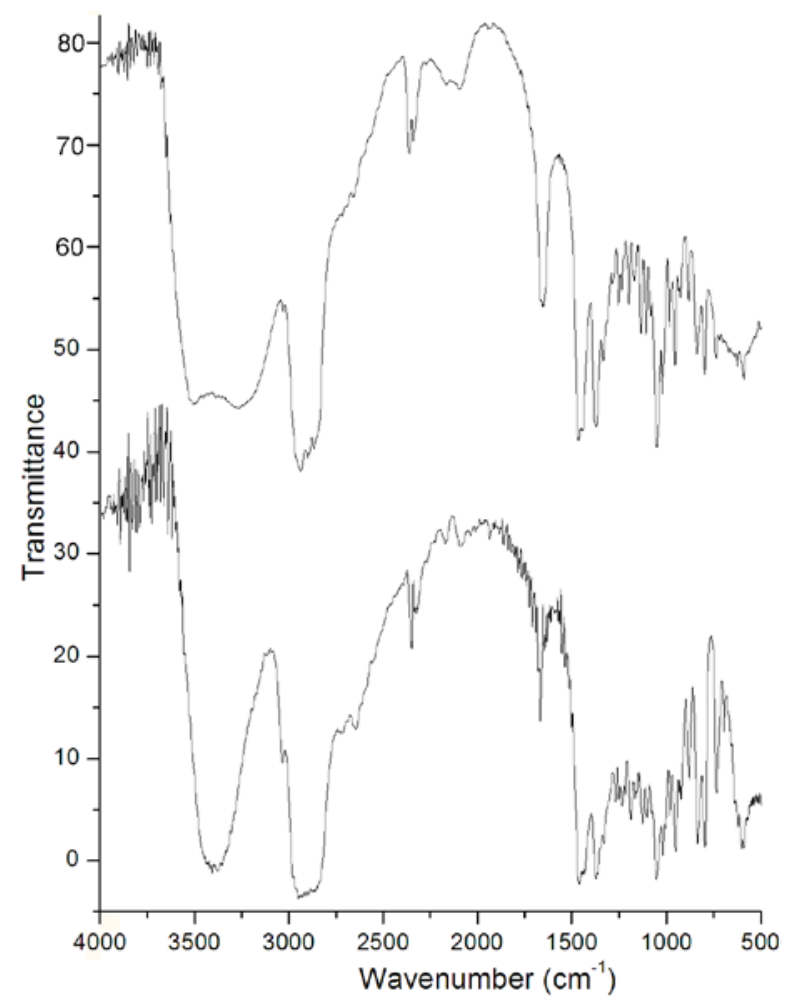

Fig.4. FT-IR spectra of the commercial cholesterol (bottom) and of uniform cholesterol particles prepared in 1-propanol/water solution (top).

In addition to previously reported findings ${ }^{26}$ wherein the effects of $\mathrm{pH}$, ionic strength, cholesterol concentration, solvent/non-solvent ratio, temperature and aging treatment were all evidenced as significant in the process of formation of uniform cholesterol platelets, the effects of various other experimental parameters on the morphology of uniform cholesterol particles were investigated. For example, centrifugation treatment and agitation with magnetically coupled stir bar do not change the morphology, but mildly distort the size uniformity of the particles, whereby the ultrasound treatment almost negligibly improves the smoothness of the particle surfaces. The particles are shown as sensitive to mild mechanical effects, as the slightest amount of manual grinding with pestle (сca. 5 - $10 \mathrm{sec}$ ) induces deviations in terms of uniformity, whereby the mere process of vacuum-assisted filtering initiates partial stacking and midly disrupts refined and well-defined character of the shape of the particles, as is shown in Fig.5. Decreasing the rate of solvent addition to $1 \mathrm{ml} / \mathrm{min}$ does not produce significant discrepancies in terms of distribution of particle shapes and sizes, although leads to occasional disruptness of the particles smoothness, which, however, becomes restored when the preparation process turns to be coupled with ultrasound agitation, as is shown in Fig.6. 


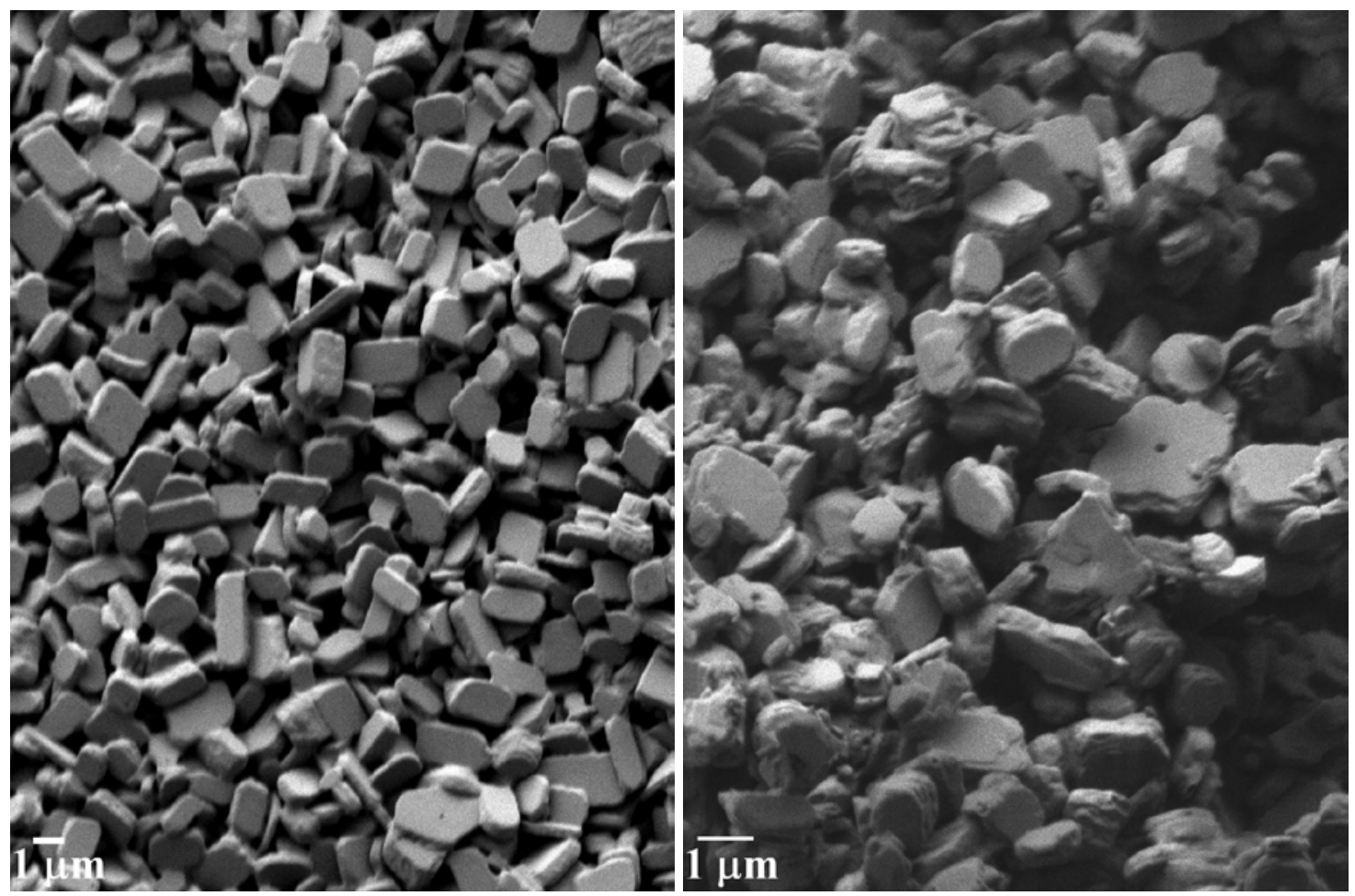

Fig.5. Cholesterol particles prepared by the procedure described in the experimental section, filtered (left), and additionally ground in mortar (right).
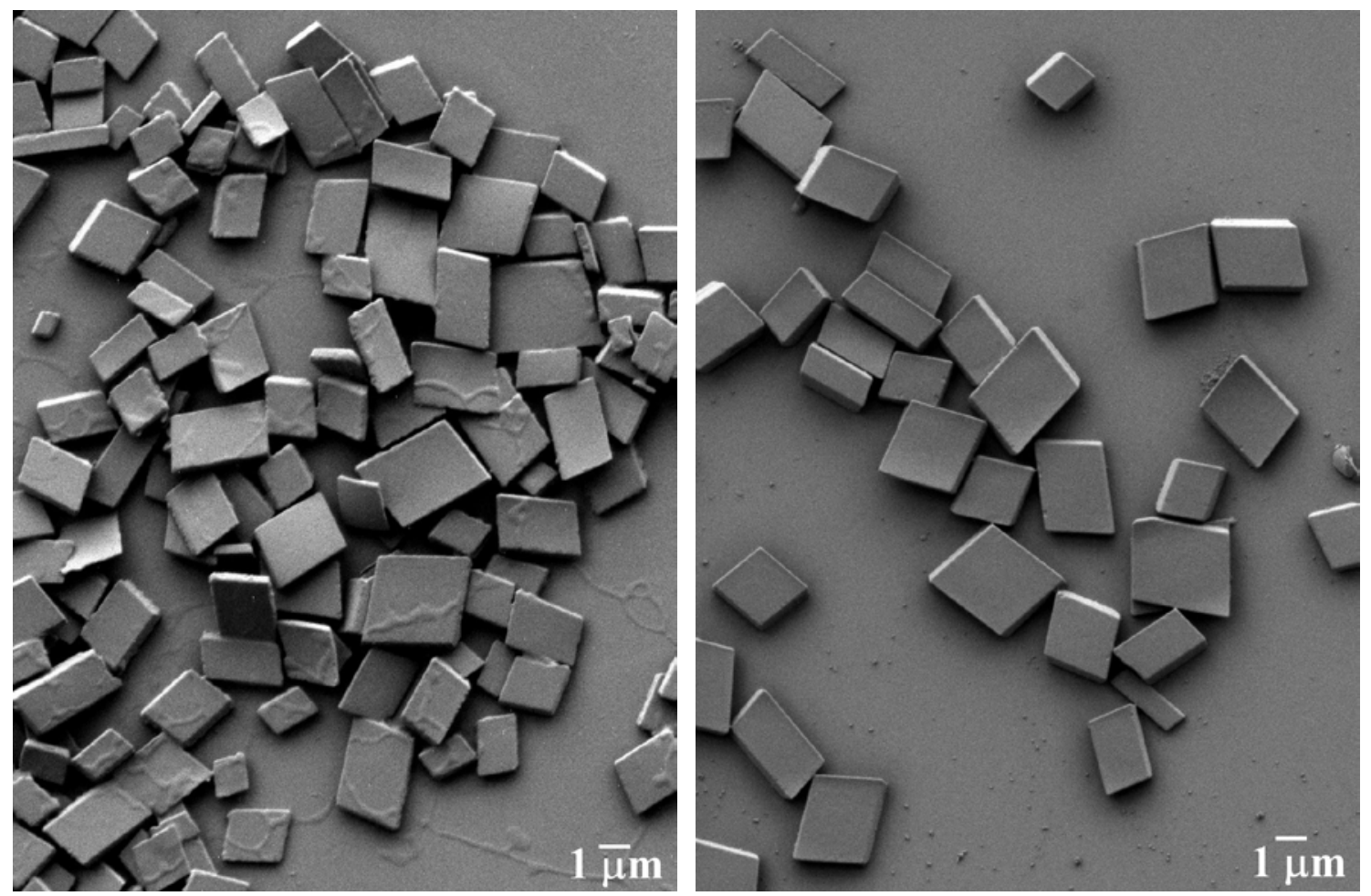

Fig.6. Cholesterol particles prepared by the procedure described in the experimental section, with the rate of non-solvent addition decreased to $1 \mathrm{ml} / \mathrm{min}$, with (right) and without (left) the influence of ultrasound. 
The following example may neatly illustrate a remarkable sensitivity of cholesterol precipitation processes on the slightest modifications introduced in the initial experimental conditions. Namely, when 2-propanol of reagent grade (99\% purity) is used as the solvent instead of 1-propanol in the described method of precipitation, slightly smaller brick-shaped particles with less pronounced edge sharpness are obtained (as a result of the increase in solubility of cholesterol with the chain length of alkanols used as solvents ${ }^{7}$ ), whereas the use of 2-propanol of technical grade (90\% purity) results in the formation of mostly needle-shaped particles, as can be seen from Fig.7.

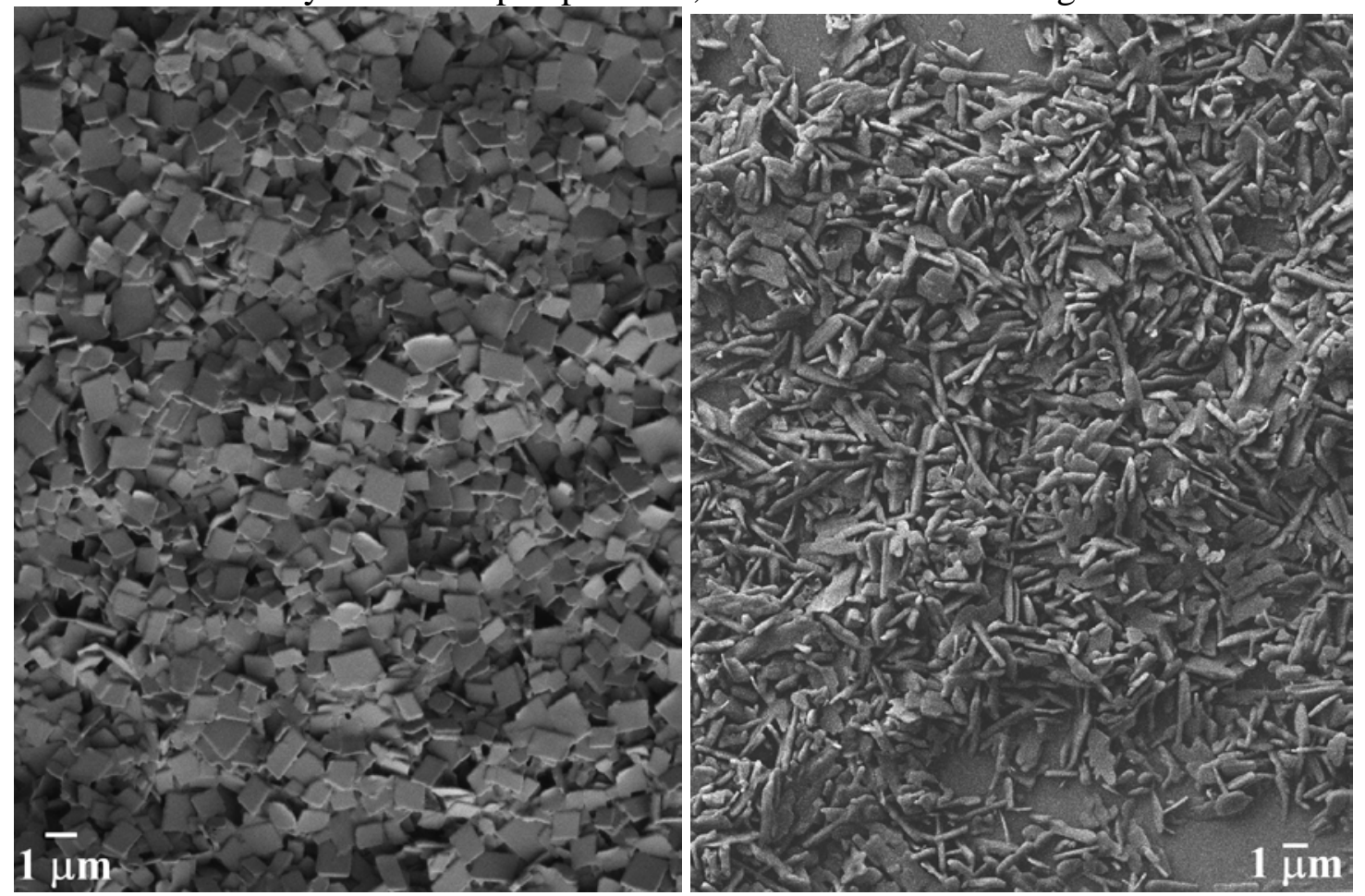

Fig.7. Cholesterol particles prepared by the procedure described in the experimental section, wherein 1propanol is substituted with 2-propanol of laboratory grade (left) and technical grade (right).

The crystallization processes were also sensitive to variations in temperature. For example, due to significant increases in solubility of cholesterol in organic solvents with temperature ${ }^{7}$, the sample prepared at $70{ }^{\circ} \mathrm{C}$ with the addition of $25 \%$ more water in comparison with the procedure described in the experimental section, yields much thinner platelets, whereas the precipitation at $31{ }^{\circ} \mathrm{C}$ with the 10 -minute aging in temperatureoscillating conditions yields partially hollow and pickered brick-shaped particles, as is shown in Fig.8. The former result coincides with similar morphologies obtained with the addition of specific additives, such as CTAB and SDS surfactants, that in the reaction medium increase the supersaturation limit of the cholesterol solutions. On the other hand, despite significant increase in solubility of cholesterol at higher salt contents (e.g. additional $5 \mathrm{ml}$ of water had to be introduced in the procedure described in the experimental section in order to induce the precipitation of cholesterol), lower electrostatic repulsion as the effect of the contracted layers of charged species around each of the dispersed particles, promotes aggregation of individual particles in the course of aging time, resulting in the formation of larger multilayered particles composed of stacked original platelets, as is shown in Fig.9. The evidenced instability of the 
dispersions of cholesterol at higher ionic strengths may be particularly interesting in the context of pathological cardiovascular states that combine high blood pressure, increased content of electrolytes in the diet and blood, and the occurence of atherosclerotic deposits of cholesterol. The effect of salt on the thickening of cholesterol particles in the precipitation stage was excluded both by unchanged particle thickness after the standard, 10 min aging time (Fig.10), and by performing the experiment that involved the same concentration of the salt, but a post-precipitation addition thereof. The latter experiment resulted in similarly pronounced aggregation during the aging stage as in the cases based on the initial addition of electrolyte, as can be evidenced from Fig.10. As a comparison, despite the fact that they also slowly undergo the process of stacking aggregation of primary platelets, the dispersions prepared in accordance with the procedure described in the experimental section remain stable and well-dispersed for extended periods of time, as is illustrated in Fig.11. In accordance with the Schulze-Hardy rule and the hydrophobic nature of cholesterol particles, the addition of electrolytes in the dispersion medium increased the rate of aggregation of individual platelets during the aging treatment, in direct proportion with concentration and valence of the introduced ionic species. Aging at increased temperatures similarly leads to an increased level of sintering of individual particles that eventually gives rise to large cholesterol blocks, which although maintain their typical rectangular morphological character. The general trend is that the larger the difference between the precipitation temperature and the aging temperature, the less time is required for the particle aggregates to attain the same level of aggregation. However, with increases in temperature during the aging treatment, the effect of dissolution of particles due to temperature-induced increases in solubility and the effect of their aggregation due to increased level of thermal movement compete and exactly at the body-temperature $\left(37^{\circ} \mathrm{C}\right.$ ) produce the most optimal conditions for comparatively fast aggregation and sintering of particles. Lower temperatures than this decrease the rate of aggregation, whereas it takes half an hour for the solid phase to get completely dissolved during aging at $40{ }^{\circ} \mathrm{C}$. Furthermore, precipitation via cooling cholesterol solutions previously heated above the supersaturation point proceeds through metastable states. This has been evidenced on numerous occasions. For example, in spite of the solution being cooled down to room temperature, precipitation processes in terms of visible formation of millisized and transparent crystals begin only after a few hours in a still system, whereas mild mixing of the mixture can induce earlier precipitation in form of smaller particles. Moreover, precipitation in accordance with the already described procedure at $31{ }^{\circ} \mathrm{C}$ yields precipitate, whereas precipitation at $34{ }^{\circ} \mathrm{C}$ does not. Parallel aging of these two dispersions at $37{ }^{\circ} \mathrm{C}$ leads to the formation of permanent precipitate in the former and, expectedly, no precipitate at all in the latter. After restoring the both suspensions to room temperature conditions, a couple of hours is required for the comprising amounts of precipitate in the two systems to equalize. On the other hand, in spite of the observed inclination of the actual processes towards metastable states, the final morphology is confirmed as independent on the solvent/non-solvent ratio prior to the addition of precipitating non-solvent, as long as the final solvent/non-solvent ratio and cholesterol concentration remain the same. 


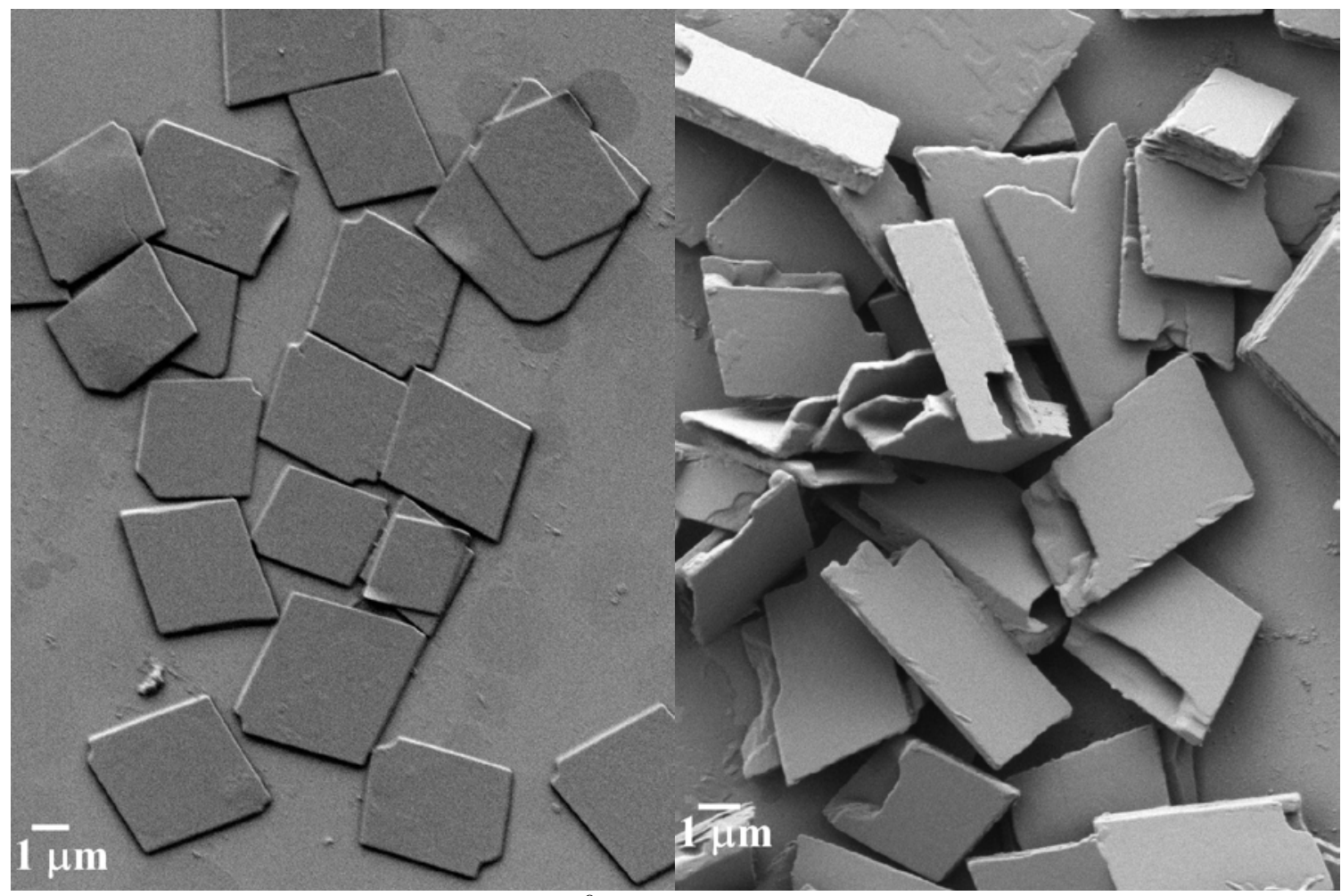

Fig.8. Cholesterol particles precipitated at $70{ }^{\circ} \mathrm{C}$ with the addition of $25 \%$ more water in comparison with the procedure described in the experimental section (left), and precipitated at $31^{\circ} \mathrm{C}$ with the subsequent 10 minute aging at the temperature-oscillating conditions in the range of $36-42{ }^{\circ} \mathrm{C}$ (right).

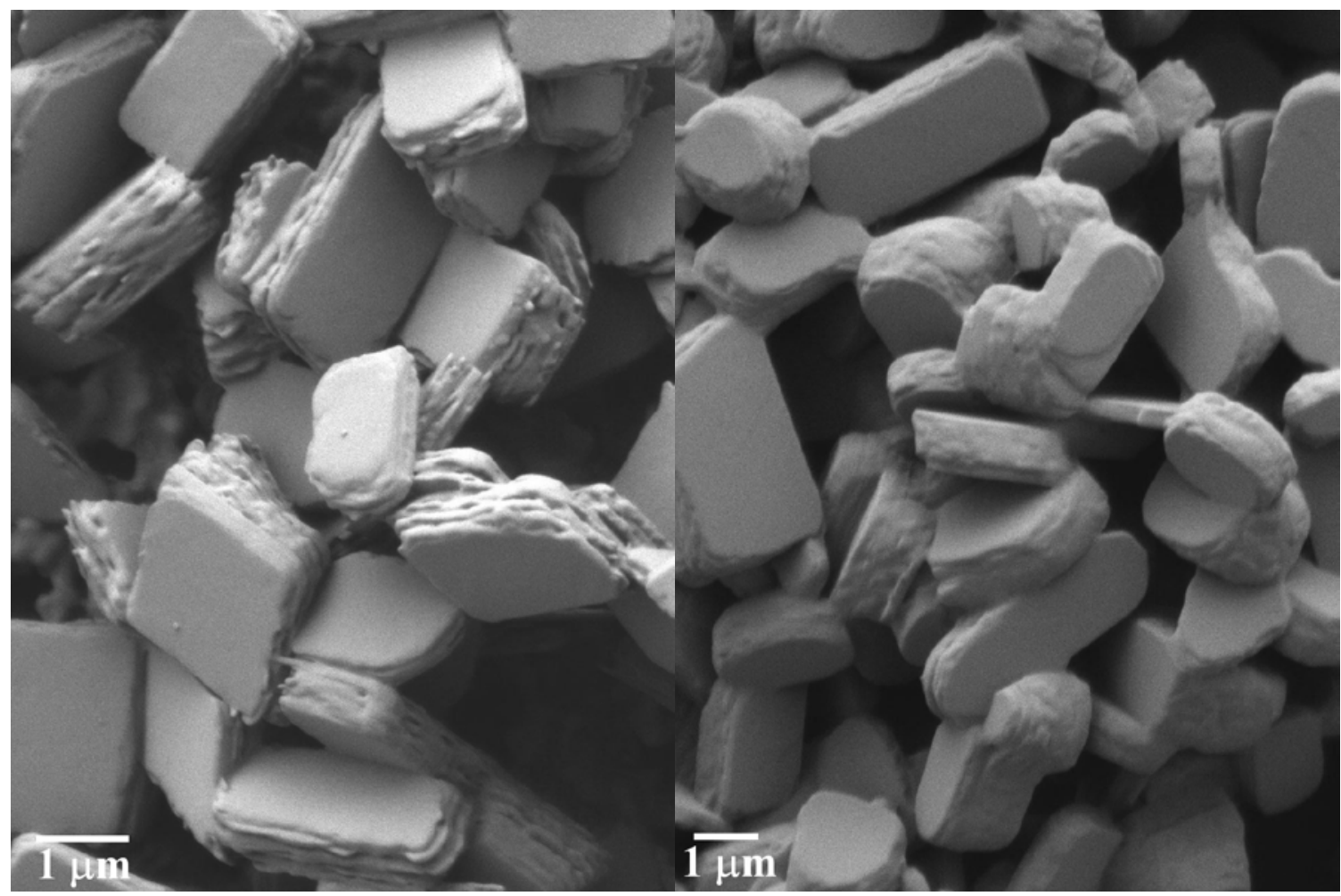


Fig.9. Cholesterol particles precipitated in accordance with the procedure described in the experimental section, but including $0.4 \mathrm{M} \mathrm{NaCl}$ in the reaction medium and $2.5 \mathrm{~h}$ aging (left), and $0.05 \mathrm{M} \mathrm{BaCl}_{2}$ with $5 \mathrm{~h}$ aging (right).

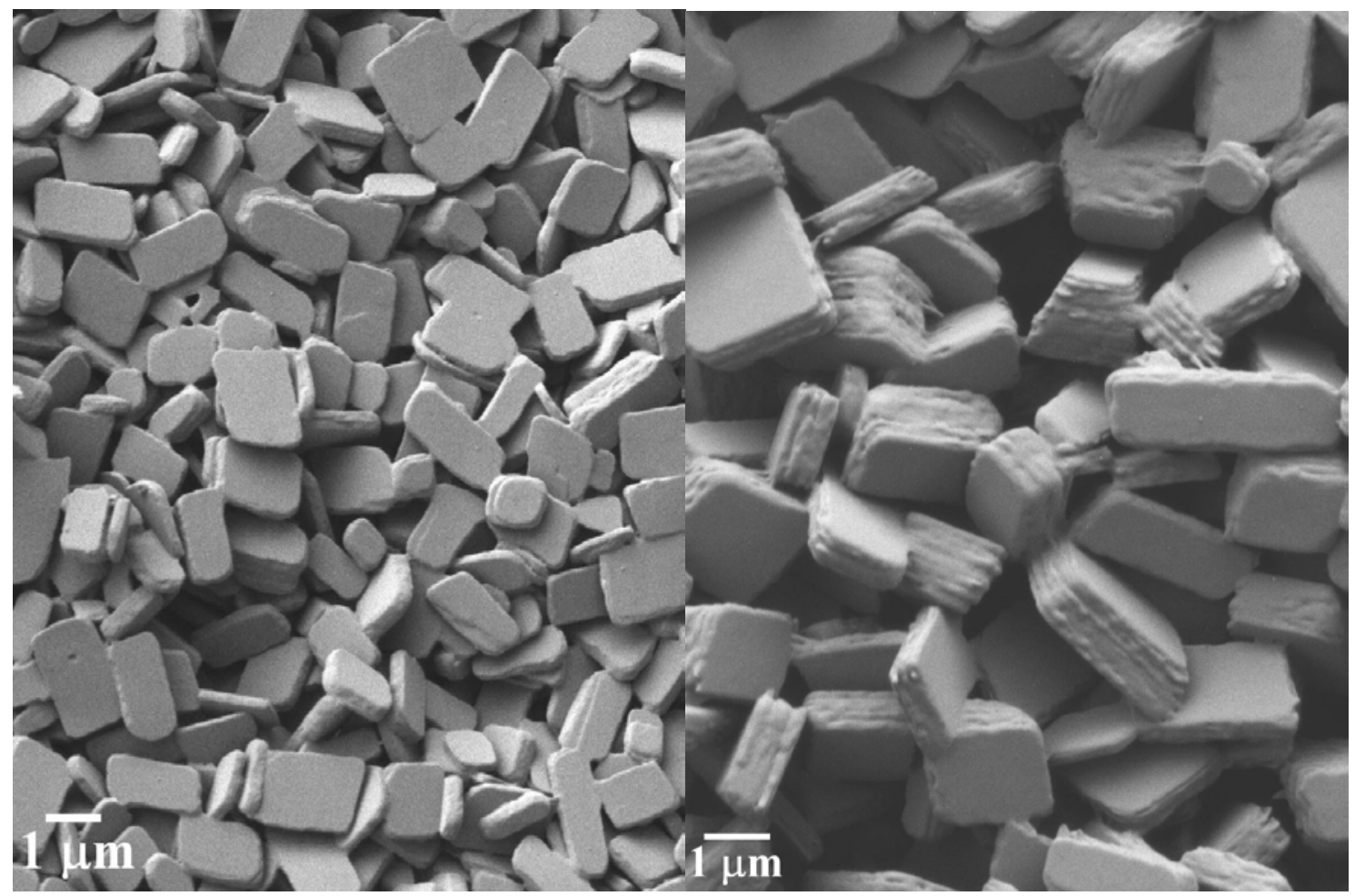

Fig.10. Cholesterol particles precipitated in accordance with the procedure described in the experimental section, but including $0.4 \mathrm{M} \mathrm{NaCl}$ in the reaction medium and 10 min aging (left), and including the late (after 10 min aging) addition of $\mathrm{NaCl}$ (resulting in $0.4 \mathrm{M}$ concentration of the salt) and $2.5 \mathrm{~h}$ aging (right).

Based on the results presented so far, it may be induced that cholesterol precipitation in simple aqueous-alcoholic environment as the one used within this work presents a process of significant sensitivity to modifications of experimental parameters of crystallization. However, on the other hand, the tendency of cholesterol crystals to adopt plate-shaped morphologies inevitably dominates the precipitation processes. This tendency is explained by the existence of stronger, H-bond intermolecular forces that link individual molecules along layers in comparison with weaker, van der Waals forces that link individual layers in the final, brick-shaped crystalline forms ${ }^{4}$. However, whereas the first stages of crystallization favor the diffusional growth of thin platelets, comparatively slow subsequent aggregation of individual platelets takes place during prolonged aging and the sedimentation phase. This proposition is supported by the visual observation of color changes in the first minute after the precipitation is initiated and the corresponding increase in thickness of individual particles, from thin leaflets to brick-shaped ones within the first 5 minutes of aging, whereby the striped cross-sections and significantly increased thickness of particles aged for longer times (up to a month) support the aggregational mechanism of the continuing growth in particle size, as is shown in Fig.11. 


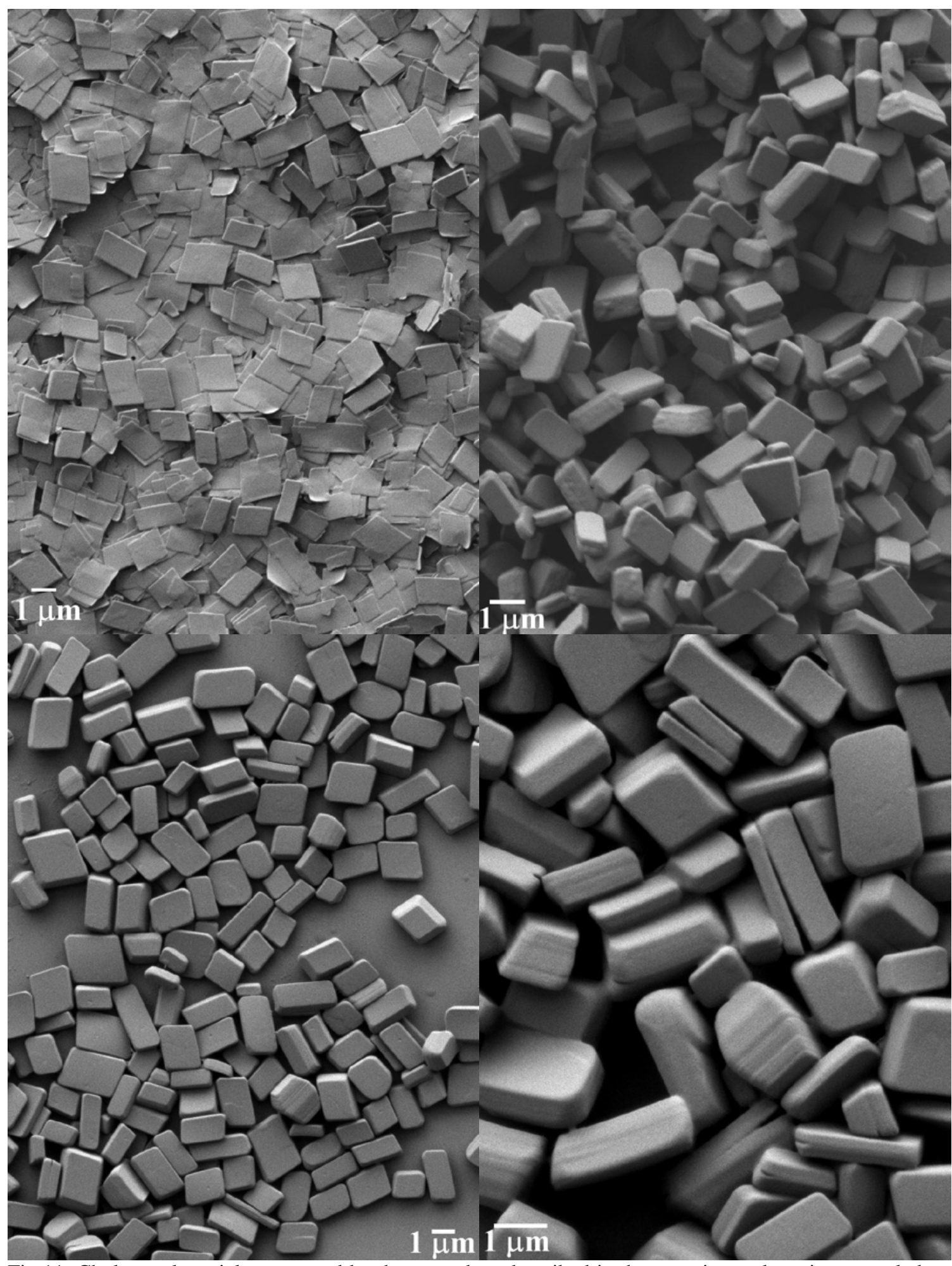

Fig.11. Cholesterol particles prepared by the procedure described in the experimental section, sampled out after 10 sec (upper left), 2 days (upper right) and one month (bottom) of aging time in an open vessel (and $500 \mathrm{ml}$ of the overall volume) at atmospheric conditions, i.e. until $95 \%$ vol. of the liquid component has evaporated. 
On one hand, the possibility of dissolution/recrystallization mechanisms (including Ostwald ripening) that might have yielded thicker particles in this case can be excluded on the basis of high uniformity of particle sizes and shapes observed in all stages of the investigated process. On the other hand, smoothness and highly refined morphological character of thick cholesterol cuboids imply an inevitable existence of mass transfer effects in the processes of particle aggregation. Because of the biological role of cholesterol as a cell membrane component, it is natural to expect its high selectivity of positioning within self-assembled structures and significant molecular recognition properties, typical of all amphiphilic biochemical compounds. Planar biomolecules with sufficiently extended $\pi$-systems that cholesterol belongs to, have, in fact, found a wide use in controlled interfacial self-assembly processes, because they tend to bond to surfaces in a flat-lying geometry ${ }^{28}$. Also, due to its relatively small head-group size (comparing to other amphiphilic components of biological membranes), a cholesterol molecule is highly movable and has the ability to quickly fill interstitial spaces, which implies its participation in increasing the order of lipid biomembranes and playing the second messenger role in signal transduction mechanisms. High selectivity of active groups within cholesterol molecules and quick rearrangements of energetically unfavorably positioned layers that come to protrude the cross-sections of stacked platelets may, therefore, explain the smoothness of the final morphologies of cholesterol particles and sediments formed by aggregation at higher temperatures or crystallization by employing complete or partial evaporation of solvent/non-solvent medium, as is shown in Fig.12. However, in contrast with the aging at room conditions, which results in stable dispersions consisting of well-defined cholesterol cuboids formed through aggregation of primary platelets grown by diffusion (Fig.11), higher aging temperatures do trigger Ostwald ripening effects and lead to significant deviations from narrow dispersity in terms of particle sizes and shapes, as can be seen from Fig.12. 


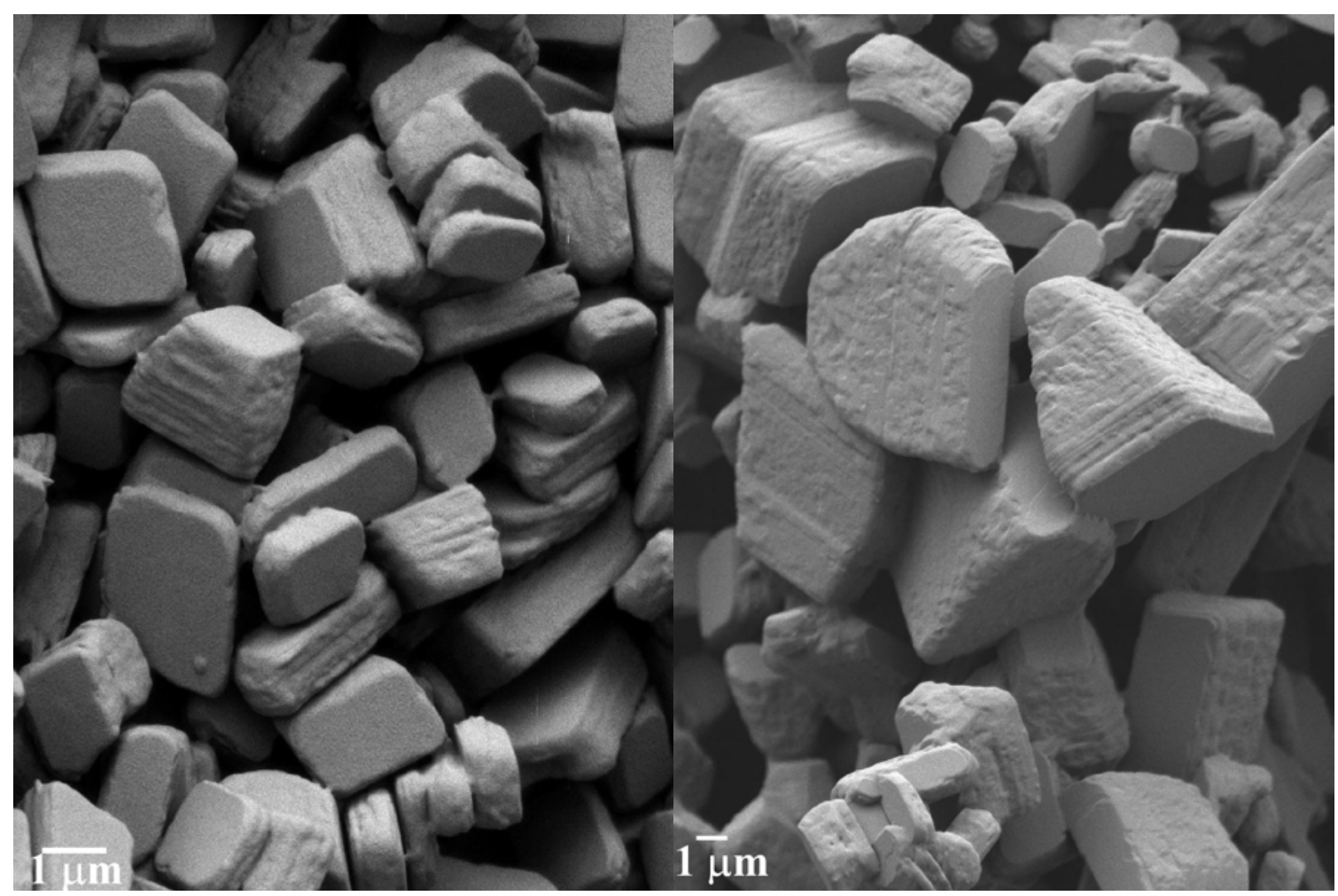

Fig.12. Cholesterol particles precipitated by the procedure described in the experimental section, but aged in a closed container for 2 days at $34{ }^{\circ} \mathrm{C}$ in an isothermal bath (left), and particles precipitated by the procedure described in the experimental section at $31{ }^{\circ} \mathrm{C}$, and aged under the same conditions as the former sample for $5 \mathrm{~h}$ at $37^{\circ} \mathrm{C}$ (right).

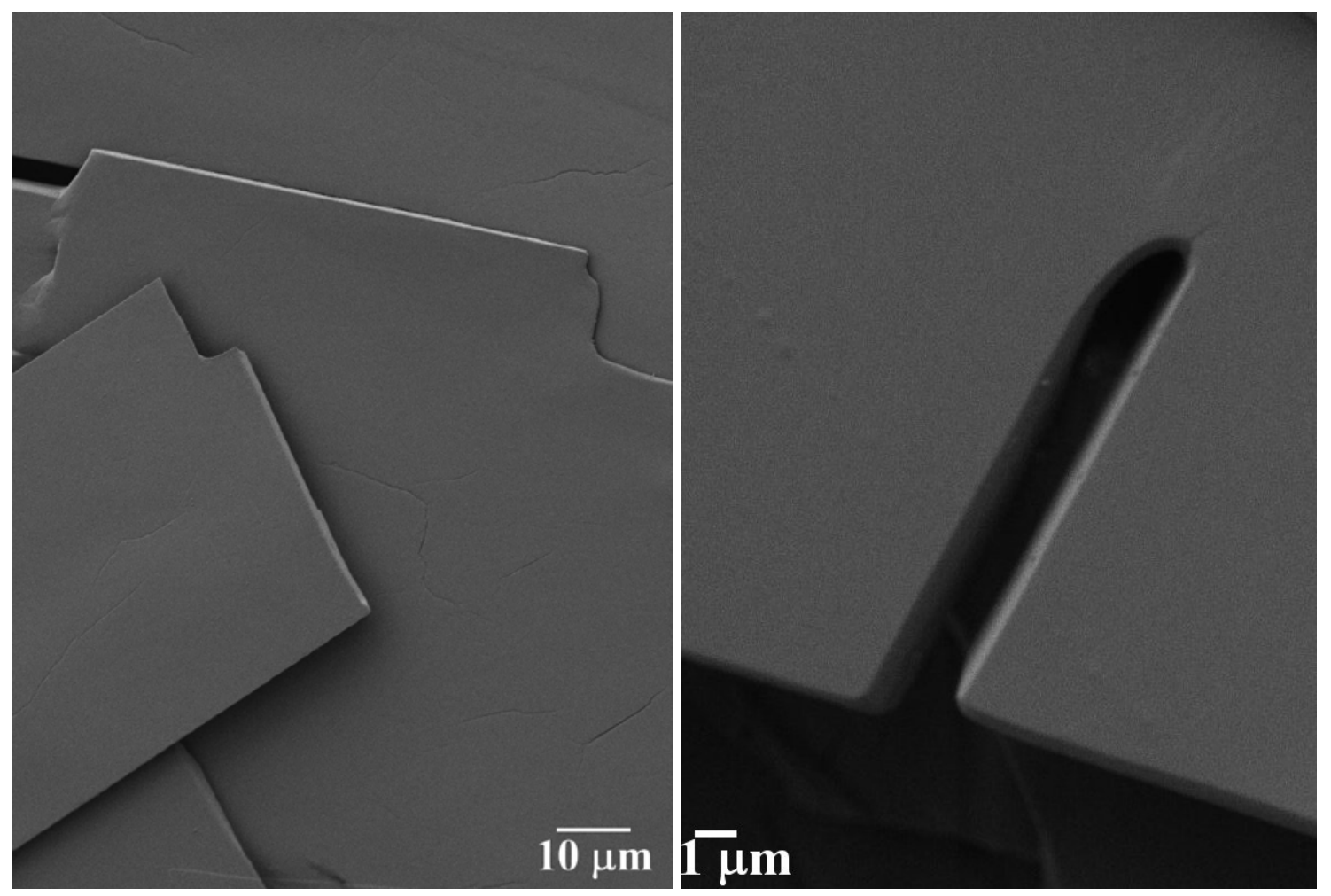


Fig.13. Cholesterol sample prepared by evaporation of solvent/non-solvent before the final 5 ml of water was introduced in the procedure; the sample was deposited in form of droplets on a watch glass, and collected upon the complete evaporation of the liquid component.

Anhydrous form is presumed to be metastable, readily transforming into monohydrate form ${ }^{4}$, whereby the action of water seems to be necessary to transform the corresponding elongated particles into plate-shaped ones. Polar 3-hydroxyl ends of cholesterol molecules form hydrogen bonds with each other and with water molecules, while each oxygen atom additionally forms three hydrogen bonds. Hydrogen-bonded network is in this case weak with water molecules having an important role in stabilizing the structure during the growth. Migration of water molecules along the tunnels that stretch in the direction of the shortest, c axis was suggested as an explanation for the ease with which crystals may change their modifications ${ }^{16}$. Water molecules and hydrogen bonds that involve inner and surface atoms may provide the crucial role in molecular recognition stages that lead to aggregation of thinner platelets into the thicker ones. Hydrogen bonds are known as strong attraction forces that vary quickly in space, enabling fast reorganization of bonded entities and remarkable molecular recognition effects $^{29}$. The fact that hydrogen bonds and van der Waals forces are involved in linking individual molecules and molecular layers in both anhydrous and monohydrate cholesterol crystal structure, as well as in interactions among individual particles, may explain high specificity of particle-particle interactions provided by the active surface groups of platelet crystals in the process of stacking and bonding aggregated platelets into precisely matching, thicker and multilayered structures.

Whereas abrupt isotropic nucleation in solution led to the formation of monodisperse platelets with 1-2 $\mu \mathrm{m}$ in size, solvent/non-solvent evaporation from cholesterol solutions with concentrations below the supersaturation limit under specific conditions resulted in the formation of millisized, flat and multilayered deposits, shown in Fig.13. Different mechanisms of crystallization are obviously present in these two approaches to precipitation of cholesterol. In contrast with the surface specificity of evaporation-induced crystallization mechanism, presumably involving gradual deposition of layers with nuclei formed in vicinity of the air-solvent interface of the drying droplet surface where the supersaturation limit becomes first exceeded, in the former approach nuclei are isotropically formed, evidently resulting in smaller and more numerous, narrowly dispersed platelets. Cholesterol is, in fact, known as an amphiphilic compound that readily positions at polar/non-polar interfaces, which is the property inherent to its major biological function as a component of cellular membranes. On the other hand, comparing to other membrane lipids, a series of fused rings provides cholesterol molecules with certain rigidity, apparently reflected on its overwhelming tendency to adopt plate-shaped crystalline morphologies. Nevertheless, selective orientation of cholesterol molecules in relatively slow crystallizing conditions and correspondingly increased diffusion path may present the reasons for the formation of fine and smooth deposits in the evaporation-induced crystallization. Whereas the precipitation induced by solvent evaporation obviously presents the evidence in favor of the reaction-limited crystal growth, the precipitation involving abrupt isotropic nucleation presents the case of diffusion-limited molecular aggregation. Comparison of the case of preparation of uniform cholesterol particles by the abrupt addition of non-solvent (described in the experimental section) and the preparation of comparatively larger, millisized cholesterol 
crystals by either evaporation of solvent/non-solvent medium or cooling of cholesterol solutions can promote the conclusion that rapid nucleation under the basic conditions used in these experiments presents an essential factor in ensuring the precipitation of monodisperse cholesterol particles.

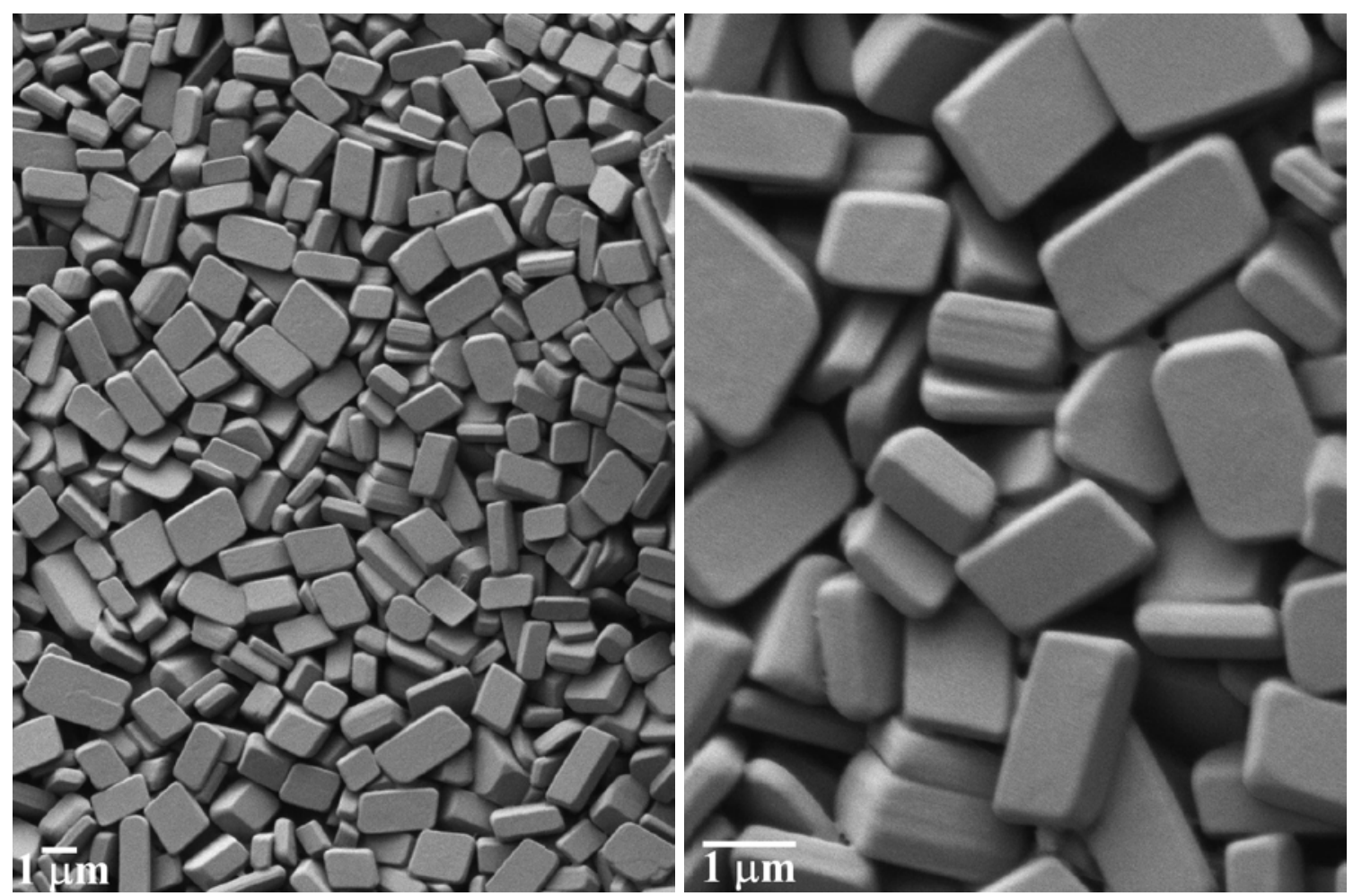

Fig.14. Cholesterol particles prepared in accordance with the procedure described in the experimental section, aged at atmospheric conditions until $95 \%$ vol. of the solvent mixture has evaporated (one month), and then sealed and kept for additional 3 months at the same conditions. 


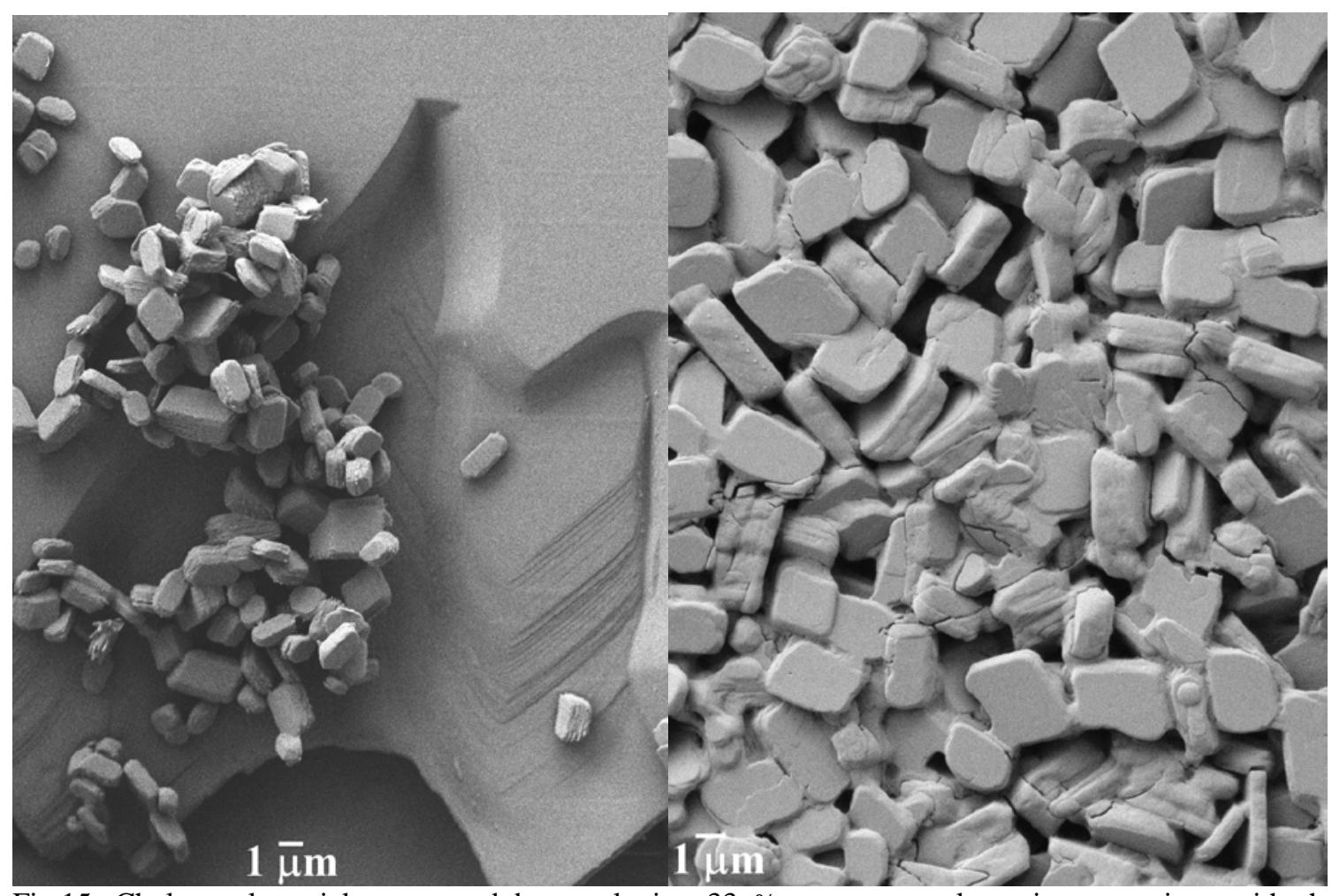

Fig.15. Cholesterol particles prepared by employing $33 \%$ more non-solvent in comparison with the procedure described in the experimental section and: 25 times larger batch size, aged for 2 days in an open container (left); aged for 2 days at $70{ }^{\circ} \mathrm{C}$ in an open container (right).

As far as the stability of cholesterol dispersions prepared herein is concerned, it may be noticed that the addition of foreign agents is not necessary to prevent permanent aggregation of well-defined platelets into large-scale multilayered deposits. The example presented in Fig.11 displays well-defined particles, formed by aggregation of smaller platelet subunits through aging for one month in an open vessel in atmospheric conditions. Evaporation of the solvent and the corresponding shift of the supersaturation limit presumably induced subtle "edification" of the existing platelets until perfectly smooth particles of partly rounded edges were obtained. These dispersed particles were stable and morphologically unchanged for months when kept in a closed vessel at atmospheric conditions, as can be seen from Fig.14. In the experiment that in addition employed $33 \%$ more non-solvent and an increased batch size, the combination of aggregation effect and solvent evaporation-induced formation of large deposits after only two days yielded large segregated deposits attached to the bottom of the vessel (Fig.15). However, increase in the aging temperature up to $70{ }^{\circ} \mathrm{C}$ upon the complete evaporation of the solvent yields not smooth, millisized deposits, but brick-shaped particles linked with the material formed by solvent evaporation (Fig.15), which is consistent with the aforementioned effects of increased temperature during the aging treatments. In this case, the evaporation process although proceeds with such a high rate that there is not enough time for aggregation processes to yield fine and smooth multilayered deposits through thermal movement and molecular recognition effects, but instead the partially aggregated platelets become linked by additionally precipitated material deposited by evaporation of the solvent at high rate. Kinetic effects, therefore, play a significant role in the processes 
of aggregation and stabilization of cholesterol suspensions. The batch and vessel size are shown to play an important role in a sense that significantly larger and smoother deposits were obtained after aging of larger batches with 25 - 100 times multiplied quantities described in the experimental procedure. This observation may be explained by both gravity effects (i.e. different pressure of the solvent column upon the multi-layered deposits formed by aggregation of primary platelets and segregating at the bottom of the vessel), and the slower rate of the shift of the supersaturation limit due to solvent evaporation and a consequently slower rate of additional precipitation of cholesterol over the course of the aging time. In addition, sensitivity of the stability of cholesterol dispersions to compositional, structural and surface charge features of the walls of the vessels that contained these colloids can be acknowledged as well. Compositionally identical dispersions may during aging result in different types of segregational behavior, depending on the surface properties of the respective containers. Epitaxial relationships and the structural co-existence of solid cholesterol phases with other crystalline materials, including phytosterols, phytostanols ${ }^{30}$, hydroxyapatite ${ }^{16}$ and other calcite phases ${ }^{13,14}$, have been previously acknowledged. Different morphological features of cholesterol deposits obtained by complete evaporation of the liquid component of the respective dispersions have correspondingly depended on the chemical nature of employed substrates (glass, $\mathrm{Cu}, \mathrm{Si}$ ). Accordingly, the stability and segregational mechanism (creaming or sedimentation) of cholesterol dispersions can be largely influenced by the surface features of the applied containers. Closely related to this observation and most important of all, charge effects may be disclosed as a crucial feature of cholesterol aggregation processes and a parameter that might be applied in control of the stability and segregation of cholesterol dispersions. In accordance with the crystallographic data, the intrinsic hydroxyl groups are expected to protrude primarily particle sides rather than their faces $^{4,16,31}$. This effect was evidenced by more pronounced charging of the particle sides by the influence of electron beam during the SEM measurements. Cholesterol platelets were also observed to aggregate in "face-to-face" manner in course of the aging time, which presents another indication of weaker electrostatic repulsion between particle faces comparing to their cross-sections. The previously reported study has correspondingly witnessed an increased level of aggregation effects at $\mathrm{pH}$ values that correspond to the isoelectric point of cholesterol particles ${ }^{26}$. Being responsible for "face-to-face" aggregation of well-defined cholesterol particles in simple solutions, electrostatic charge effects might be consequently considered as an important physicochemical factor in potential prevention of in vivo segregation and deposition of fine cholesterol particles.

\section{Conclusions}

As a complementary continuation of the previous report on the investigation of cholesterol precipitation processes, additional subtle effects, including non-solvent addition rate, temperature, solvent purity, aging treatments, ultrasound agitation and fine mechanical effects, altogether with a comprehensive discussion of the mechanisms of cholesterol precipitation, are presented in this work. Morphological sensitivity of cholesterol precipitates in relation to all of the mentioned effects can be acknowledged, although biaxially-grown particles were formed in each of the imposed experimental conditions of precipitation. Each of the aforementioned observations of sensitivity of the 
dispersed cholesterol particles upon the conditions of their formation and aging may present a starting point for more comprehensive and refined studies of the structural and aggregational nature of solid cholesterol. Nevertheless, it has been shown that the longterm stability of suspensions of well-defined and uniform cholesterol particles in simple aqueous-alcoholic environment can be achieved, without the presence of any additives. The promotion of enormously simple and eco-friendly method for the preparation of uniform and well-defined cholesterol particles such as the one presented in this work may provide improved understanding of the behavior of solid cholesterol in liquid

environments and, thereupon, present a step forward towards a simpler and more elegant manipulation of the biological discrepancies that involve its presence.

\section{References:}

1. J. H. Richards, J. P. Hendrickson - "The Biosynthesis of Steroids, Terpenes, and Acetogenins", W. A. Benjamin, New York/Amsterdam (1964).

2. F. R. Maxfield, I. Tabas - "Role of Cholesterol and Lipid Organization in Disease", Nature 438, $612-21$ (2005).

3. H. S. Shieh, L. G. Hoard, C. E. Nordman - "Crystal Structure of Anhydrous Cholesterol”, Nature 267, $287-9$ (1977).

4. R. S. Abendan, J. A. Swift - "Surface Characterization of Cholesterol Monohydrate Single Crystals by Chemical Force Microscopy”, Langmuir 18, 4847 - 4853 (2002).

5. A. Elizabeth, C. Joseph, M. A. Ittyachen - "Growth and Micro-Topographical Studies of Gel Grown Cholesterol Crystals”, Bulletin of Materials Science 24 (4) 431 - 434 (2001).

6. N. Garti, L. Karpuj, S. Sarig - "Correlation between Crystal Habit and the Composition of Solvated and Nonsolvated Cholesterol Crystals”, Journal of Lipid Research 22, 785 - 791 (1981).

7. G. L. Flynn, Y. Shah, S. Prakongpan, K. H. Kwan, W. I. Higuchi, A. F. Hofmann - "Cholesterol Solubility in Organic Solvents”, Journal of Pharmaceutical Sciences 68 (9) 1090 - 1097 (1979).

8. R. J. Jandacek, M. R. Webb, F. H. Mattson - "Effect of an Aqueous Phase on the Solubility of Cholesterol in an Oil Phase”, Journal of Lipid Research 18, 203 - 210 (1977).

9. S. N. Kalkura, S. Devanarayanan - "Growth of Cholesterol Crystals in Silica Gel", Journal of Materials Science Letters 5, 741 - 741 (1986).

10. P. A. Monzon, E. D. Siebert - "The Effect of Electrolytes on Cholesterol Crystal Growth in a Dynamic System”, Book of Abstracts, 215 ${ }^{\text {th }}$ ACS National Meeting, Dallas, March 29 - April 2 (1998).

11. J. Ahumada, E. D. Siebert - "Cholesterol Crystal Growth from a Dynamic and Static Solvent System”, Book of Abstracts, $211^{\text {th }}$ ACS National Meeting, New Orleans, LA, March 24-28 (1996).

12. N. M. Sandarac, M. Ashok, N. Kalkura - "Observation of Cholesterol Nucleation in a Magnetic Field”, Acta Crystallographica D58, 1711 - 1714 (2002).

13. M. C. Frincu, S. D. Fleming, A. L. Rohl, J. A. Swift - "The Epitaxial Growth of Cholesterol Crystals from Bile Solutions on Calcite Substrates", Journal of the American Chemical Society 126, 7915 - 7924 (2004).

14. X. Liao, T. S. Wiedmann - »Formation of Cholesterol Crystals at a Mucin Coated Substrate«, Pharmaceutical Research 23 (10) 2413 - 2416 (2006).

15. M. C. Frincu, R. E. Sharpe, J. A. Swift - "Epitaxial Relationships between Cholesterol Crystals and Mineral Phases: Implication for Human Disease”, Crystal Growth \& Design 4 (2) 223 - 226 (2004).

16. B. M. Craven - “Crystal Structure of Cholesterol Monohydrate”, Nature 260, 727 - 9 (1976).

17. B. E. Cham - "Plaque Cholesterol and Calcium: the Value of EBCT in the Detection of Coronary Artherosclerosis”, European Journal of Clinical Investigation 31 (6), 467 - 468 (2001).

18. D. F. Laird, M. R. Mucalo, Y. Yokogawa - "Growth of Calcium Hydroxyapatite (Ca-HAp) on Cholesterol and Cholestanol Crystals from a Simulated Body Fluid: A Possible Insight into the Pathological Calcifications Associated with Artherosclerosis", Journal of Colloid and Interface Science 295, 348 - 363 (2006). 
19. T. Nishioka, S. Tazuma, G. Yamashita, G. Kajiyama - "Quantitative Assessment of Comparative Potencies of Cholesterol-Crystal-Promoting Factors: Relation to Mechanistic Characterization”, Biochemical Journal 332, 343 - 350 (1998).

20. T. Nishioka, S. Tazuma, G. Yamashita, G. Kajiyama - "Partial Replacement of Bile Salts Causes Marked Changes of Cholesterol Crystallization in Supersaturated Model Bile Systems", Biochemical Journal 340, 445 - 451 (1999).

21. K. J. V. Erpecum, P. Portincasa, M. Gadella, B. J. M. V. De Heijning, G. P. V. B. Henegouwen, W. Renooij - "Effects of Bile Salt Hydrophobicity on Crystallization of Cholesterol in Model Bile”, European Journal of Clinical Investigation 26 (7) 602 (1996).

22. N. T. Saraswathi, F. D. Gnanam - "Effect of Medicinal Plants on the Crystallization of Cholesterol”, Journal of Crystal Growth 179, 611 - 617 (1997).

23. R. M. Epand, D. W. Hughes, B. G. Sayer, N. Borochov, D. Bach, E. Wachtel - "Novel Properties of Cholesterol-Dioleoylphosphatidylcholine Mixtures”, Biochimica et Biophysica Acta 1616, 196 -208 (2003).

24. J. Ahumada, E. D. Siebert - "Cholesterol Crystal Growth from a Dynamic and Static Solvent System”, Book of Abstracts, 213 ${ }^{\text {th }}$ ACS National Meeting, San Francisco, April 13-17 (1997).

25. L. Christiansen, M. Karjalainen, T. Seppanen-Laakso, R. Hiltunen, J. Yliruusi - "Effect of $\beta$ Sitosterol on Precipitation of Cholesterol from Non-Aqueous and Aqueous Solutions", International Journal of Pharmaceutics 254 (2) 155 - 166 (2003).

26. V. Uskoković, E. Matijević - "Uniform Particles of Pure and Silica Coated Cholesterol”, Journal of Colloid and Interface Science 315 (2) 500 - 511 (2007).

27. C. R. Loomis, G. G. Shipley, D. M. Small - "The Phase Behavior of Hydrated Cholesterol", Journal of Lipid Research 20, 525 - 535 (1979).

28. J. V. Barth, G. Costantini, K. Kern - "Engineering Atomic and Molecular Nanostructures at Surfaces”, Nature 437, 671 - 679 (2005).

29. D. Chandler - "Interfaces and the Driving Force of Hydrophobic Assembly", Nature 437, 640 647 (2005).

30. S. M. Mel'nikov, J. W. M. S. ten Hoorn, B. Bertrand - "Can Cholesterol Absorption be Reduced by Phytosterols and Phytostanols via a Cocrystallization Mechanism?”, Chemistry and Physics of Lipids 127, 15 - 33 (2004).

31. R. S. Abendan, J. A. Swift - "Dissolution on Cholesterol Monohydrate Single-Crystal Surfaces Monitored by in Situ Atomic Force Microscopy”, Crystal Growth \& Design 5, 2146 - 53 (2005). 